\title{
ASOCIACIÓN ENTRE VIOLENCIA CONTRA LA MUJER EJERCIDA POR LA PAREJA Y DESNUTRICIÓN CRÓNICA EN SUS HIJOS MENORES DE CINCO AÑOS EN PERÚ
}

\author{
Paulo Ruiz-Grosso ${ }^{1,2, a}$, Christian Loret de Mola ${ }^{1,3,4 ~ b}, \mathrm{~J}$. Jaime Miranda $2,4, \mathrm{c}$
}

\begin{abstract}
RESUMEN
Objetivos. Evaluar la asociación entre violencia contra la mujer ejercida por su pareja y la prevalencia de desnutrición crónica en sus hijos menores de cinco años. Materiales y métodos. Análisis secundario transversal de la Encuesta Demográfica y de Salud Familiar (ENDES) del año 2011, realizada en el Perú y con representatividad nacional. La variable dependiente fue desnutrición crónica en el último hijo menor de cinco años. Las variables independientes fueron el autorreporte de violencia emocional, violencia física (leve o severa) y violencia sexual, ejercidas por el último compañero o esposo de la madre en los últimos 12 meses. Realizamos análisis univariados y multivariables utilizando modelos generalizados lineales (regresión log-binomial), teniendo en cuenta la naturaleza compleja de la muestra. Se ajustaron los modelos para variables sociodemográficas y de salud madre hijo. Se calcularon razones de prevalencias (RP) e intervalos de confianza al 95\%. Resultados. Se analizaron datos de 5154 madres e hijos. La prevalencia de desnutrición crónica fue de 19,8\% (IC 95\%: 18,1-21,6). En el análisis multivariable se encontró una mayor prevalencia de desnutrición crónica en los hijos de madres con historia de violencia física severa RP 1,27\% (IC 95\%: 1,09-1,48). No hubo evidencia de asociación entre la prevalencia de desnutrición crónica y los otros tipos de violencia ejercida por la pareja. Conclusiones. El reporte de violencia física severa en el último año, por parte de las madres, estuvo asociado a un aumento en la prevalencia de desnutrición crónica en sus niños menores de cinco años.
\end{abstract}

Palabras clave: Violencia contra la mujer; Trastornos de la nutrición del niño; Desnutrición; Niño (fuente: DeCS BIREME).

\section{ASSOCIATION BETWEEN VIOLENCE AGAINST WOMEN INFLICTED BY THE PARTNER AND CHRONIC MALNUTRITION IN THEIR CHILDREN UNDER FIVE YEARS OLD IN PERU}

\begin{abstract}
Objectives. To assess the association between violence against women inflicted by their partner and the prevalence of chronic malnutrition in their children less than five years old. Materials and methods. A secondary cross-sectional analysis of the 2001 Peru Demographic and Health Survey performed in Peru, with national representativeness. The dependent variable was chronic malnutrition in the youngest child under five years old. The independent variables were emotional violence, physical violence (mild or severe) and sexual violence self-report; the violence was inflicted by the mother's last partner or spouse in the last 12 months. Univariate and multivariate analysis was performed, generalized linear models (log-binomial regression) were used, and the nature of the sample was taken into account. Models for socio-demographic and mother-son health variables were adjusted. Prevalence ratios (PR) and confidence intervals were calculated. Results. Data from 5,154 mothers and children were analyzed. The prevalence of chronic malnutrition was 19.8\% (Cl 95\%: 18.1-21.6). In the multivariate analysis, a higher prevalence of chronic malnutrition was found in the children whose mothers had a history of severe physical violence history: PR=1.27\% (IC $95 \%$ : 1.09-1.48). There was no evidence of association between the prevalence of chronic malnutrition and other kinds of violence inflicted by a partner. Conclusions. The mothers' report of physical violence in the last year was associated with an increased prevalence of chronic malnutrition in children under five years old.
\end{abstract}

Key words: Violence against women; Child nutritional disorders; Malnutrition; Child (source: MeSH NLM).

\footnotetext{
Grupo de Trabajo en Salud Mental, Universidad Peruana Cayetano Heredia. Lima, Perú.

CRONICAS Centro de Excelencia en Enfermedades Crónicas, Universidad Peruana Cayetano Heredia. Lima, Perú.

Programa de Pós Graduação em Epidemiologia, Centro de Pesquisas Epidemiológicas, Universidade Federal de Pelotas, Pelotas, Brasil.

Facultad de Medicina, Universidad Peruana Cayetano Heredia. Lima, Perú.

Médico cirujano; ${ }^{\mathrm{b}}$ médico, magíster en Epidemiología; ${ }^{\mathrm{c}}$ médico, magíster y doctor en Epidemiología

Recibido: 05-08-13 Aprobado: 20-11-13
}

Citar como: Ruiz-Grosso P, Loret de Mola C, Miranda JJ. Asociación entre violencia contra la mujer ejercida por la pareja y desnutrición crónica en sus hijos menores de cinco años en Perú. Rev Peru Med Exp Salud Publica. 2014;31(1):16-23. 


\section{INTRODUCCIÓN}

En el Perú y el mundo existen aún concepciones culturales patriarcales que crean inequidades de género $y$, consecuentemente, propician la violencia contra la mujer, la cual muchas veces se desarrolla dentro del mismo hogar (1). La violencia doméstica contra la mujer, que puede ser propiciada por cualquier miembro del hogar, así como la ejercida específicamente por la pareja íntima, puede ser clasificada de manera general en tres grupos: física, sexual o emocional, siendo que las prevalencias de sufrir algún tipo de estas oscilan entre el 20 y $60 \%$ en diferentes países de Latinoamérica y del mundo, incluyendo el Perú, donde la prevalencia de violencia contra la mujer ejercida por la pareja íntima, foco del siguiente estudio, llega al $61 \%$ en algunas provincias ${ }^{(2-4)}$.

La violencia contra la mujer puede influenciar en el estado nutricional de sus hijos a través de factores tanto biológicos, conductuales, sociales y/o contextuales ${ }^{(5)}$. Las personas que sufren de algún tipo de violencia, ya sea física o emocional, son más propensas a sufrir de algún desorden o enfermedad mental (6-8), tienen peores índices de calidad de vida y una pobre sensación de bienestar de salud general ${ }^{(9)}$, lo cual puede afectar negativamente tanto la crianza, como el estado nutricional de la madre y el niño. Además, existe evidencia de que los hijos de mujeres que experimentan episodios de violencia doméstica presentan bajo peso al nacer y parto pretérmino ${ }^{(10-13)}$, lo cual, a su vez, se ha visto relacionado con problemas nutricionales en los primeros años de vida ${ }^{(14)}$.

Si bien vemos que existe plausibilidad para pensar en una asociación entre violencia contra la mujer ejercida por la pareja y desnutrición en sus hijos, son pocos los estudios que han explorado esta relación y en escenarios diferentes al latinoamericano. Por tanto, es el objetivo del presente estudio evaluar si existe una asociación entre violencia contra la mujer ejercida específicamente por la pareja íntima y la prevalencia de desnutrición crónica en sus hijos menores de cinco años, en un contexto latinoamericano.

\section{MATERIALES Y MÉTODOS}

\section{DISEÑO DEL ESTUDIO}

Estudio transversal usando la Encuesta Demográfica y de Salud Familiar (ENDES) del año 2011, llevada a cabo por el Instituto Nacional de Estadística e Informática del Perú. La ENDES es una encuesta continua anual con muestreo probabilístico, autoponderado, estratificado, multietápico e independiente para cada departamento del Perú. Detalles sobre el tipo de encuesta y sus características han sido publicados previamente ${ }^{(15)}$.

\section{POBLACIÓN DE ESTUDIO}

La ENDES incluye una muestra aleatoria, representativa a nivel nacional, de mujeres entre 15 y 49 años de edad residentes habituales de los hogares elegidos, y sus hijos menores de cinco años. Residencia habitual fue definida como haber pernoctado en la vivienda la noche anterior al día de la entrevista.

Para el análisis se tomaron datos de madres y su último hijo menor de cinco años al momento de la entrevista, en caso tuviese más de uno, para evitar tener observaciones correlacionadas entre hermanos de la misma madre. Es importante resaltar que los datos de violencia solo se tomaron a una subpoblación (muestra aleatoria) del total de mujeres entrevistadas. Los criterios de selección, incluyendo la selección de información correspondiente a las observaciones madre-hijo se muestran en la Figura 1.

\section{VARIABLE DEPENDIENTE}

La variable dependiente fue "desnutrición crónica", la cual fue obtenida para el último hijo menor de cinco años. Las medidas de talla fueron obtenidas usando tallímetros calibrados y estandarizados, se midieron a los niños menores de dos años en posición de cubito dorsal, y los mayores en pie. Se definió desnutrición crónica como tener un valor $Z$ de talla para edad con dos desviaciones estándar (DE) por debajo de la media para su edad ${ }^{(16)}$.

\section{VARIABLES INDEPENDIENTES}

Las variables independientes fueron los tipos de violencia que la mujer podría haber experimentado alguna vez en los últimos 12 meses, todas infringidas por la pareja más reciente, específicamente esposo o compañero. La

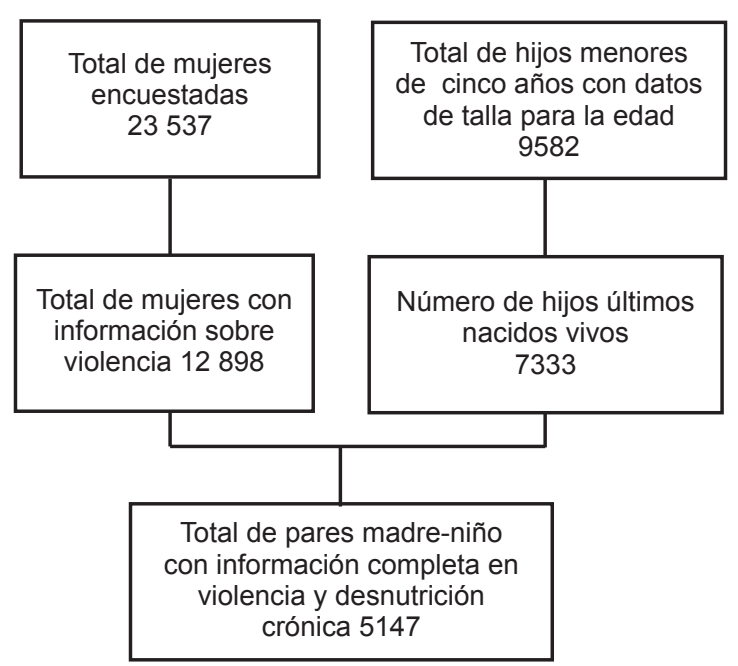

Figura 1. Diagrama de inclusión de participantes en el análisis 
encuesta preguntó, para todos los tipos de violencia, si la mujer había sufrido episodios muchas veces, alguna vez o nunca. Se consideró como caso de violencia las mujeres que respondieron alguna vez o muchas veces a las preguntas sobre tipos de violencia. Las definiciones operacionales de estas variables fueron las siguientes. Violencia emocional: si la entrevistada reportó haber sido amenazada o humillada verbalmente. Violencia sexual: si se utilizó fuerza física para obligar a mantener relaciones sexuales o fue obligada a realizar actos sexuales que no aprobaba. Violencia física: fue categorizada en solo leve, cuando se reportó haber experimentado empujones, palmazos, golpes con el puño y/o patada, y severa, cuando se reportó haber sufrido de intento de estrangulamiento, amenaza con cuchillos y/o heridas con cuchillos, independientemente de si reportaron, además, violencia leve.

\section{VARIABLES CONFUSORAS}

Se evaluaron como posibles factores de confusión a las variables sociodemográficas del hogar: residencia en zona rural o urbana, índice de riqueza (expresado en quintiles de muy pobre a muy rico) y edad del jefe del hogar; de la madre: edad, nivel educativo máximo alcanzado (sin educación, primaria, secundaria o superior), uniones conyugales (una o más de una), si recibió agresión durante el embarazo (por cualquier persona, no solo la pareja actual), haber agredido a la pareja (esposo) en ausencia de agresión; madre trabaja y si lo hace fuera de casa; de la última pareja: nivel educativo máximo alcanzado, frecuencia con que se embriaga, edad y residencia en la misma casa; y del hijo analizado: edad, sexo, orden de nacimiento, peso al nacer (kilogramos), tiempo de gestación al primer control, número de visitas prenatales, lugar de parto, parto profesional (atendido por médico, enfermera u obstetra), control posnatal dentro de los dos primeros meses de nacido, duración de la lactancia y uso de biberón. Estas variables fueron elegidas basadas en los modelos de regresión utilizados. Todas las variables incluidas alteraban significativamente la varianza del modelo para cada tipo de violencia, por lo que se optó por mantenerlas dentro de cada uno de los modelos analizados. Todas las variables de confusión fueron autorreportadas por la madre en el momento de la entrevista. La variable frecuencia con la que la pareja se embriaga no fue incluida en los modelos multivariables por su pobre tasa de respuesta en nuestra submuestra (<75\%), con pérdida de información no aleatoria que podría estar relacionada a violencia ejercida por la pareja y desnutrición crónica, lo cual podría sesgar nuestros resultados.

\section{ANÁLISIS ESTADÍSTICO}

Para el análisis estadístico se utilizó Stata versión 11 (StataCorp LP, College Station, TX, USA). En las estimaciones se incluyeron ponderaciones que tienen en cuenta el diseño complejo y multietápico de la ENDES 2011. Así, tanto los análisis descriptivos como los modelos univariados y multivariables se realizaron usando el comando svy para muestras complejas. Es importante resaltar que la ENDES tiene más de 23 mil observaciones, que incluye mujeres solteras y/o sin hijos, por tanto, los análisis finales fueron hechos en una subpoblación solo con mujeres con datos de violencia, hijos y compañero. Para esto se utilizó la opción "subpop" del programa STATA durante el análisis de regresión, para incluir en el análisis final solo las observaciones que tenían todos los datos necesarios para el análisis, considerando ponderaciones de toda la muestra, calculando así correctamente los errores estándar de las diferentes regresiones.

La descripción de las variables categóricas se realizó utilizando frecuencias, mientras que para las numéricas se usaron medidas de tendencia central y dispersión. Para los análisis univariado y multivariable se utilizó un modelo lineal generalizado (regresión log-binomial), similar a regresión logística, solo que en lugar de calcular odds ratios (OR) se calcularon razones de prevalencia (RP) y sus intervalos de confianza al 95\% (IC 95\%). Esta elección fue tomada debido a que la frecuencia del evento resultado, desnutrición crónica, era mayor al $5 \%$ y los OR podrían sobrestimar la magnitud de la asociación (17).

No se incluyeron todos los tipos de violencia en un mismo modelo de regresión, por la posibilidad de colinearidad, optándose por realizar una regresión individual para cada tipo de violencia. En cada regresión, para elegir las variables confusoras que entrarian en los modelos finales, se usó la técnica Stepwise forward \& backward, así se incluyeron y quitaron las variables de confusión secuencialmente, hacia adelante (comenzando con un modelo vacío) y hacia atrás (con modelo lleno), basados en la significancia de cada variable confusora para el modelo de regresión, hasta quedarse con un modelo individual para cada tipo de violencia y sus variables de confusión más relevantes estadísticamente. Se mantuvo en cada modelo las variables con un $p<0,2$.

Dado que los datos de violencia fueron tomados solo de una submuestra de mujeres, se hicieron análisis univariados para comparar las diferencias entre las madres que fueron excluidas y las que no, en términos de las covariables de madre e hijo.

\section{ASPECTOSÉTICOS}

El presente es un estudio que se basa en análisis secundarios, los cuales fueron realizados utilizando datos de una base de dominio público (ENDES del año 2011), en la cual no se consigna información que permita conocer la identidad de los participantes ni la ubicación de sus hogares. 


\section{RESULTADOS}

La base de datos de la ENDES 2011 contiene información de 23537 mujeres y 9582 niños menores de cinco años; 12898 mujeres tenían información sobre violencia y 7333 reportaron tener al menos un hijo de cinco años y este contaba con datos de talla para edad, de estas, 2186 no tenía datos sobre violencia. En total se analizaron datos de 5147 madres y su último hijo de cinco años (Figura 1).

Al comparar las madres con datos de desnutrición incluidas en el análisis final y las que no, vimos que las incluidas tenían una edad media mayor en 1,16 años $(p<0,001)$, mayor porcentaje de ruralidad $(p<0,001)$, una tendencia a tener menor índice de riqueza $(p<0,001)$ y escolaridad $(p<0,001)$. Los hijos no mostraron diferencias en la edad media $(p=0,443)$, pero sí en las prevalencias de desnutrición crónica:
19,8\% (IC 95\%: 18,1-21,6) en aquellos incluidos versus $15,7 \%$ (IC 95\%: 13,2-18,2) en los no incluidos en el análisis.

La prevalencia total de desnutrición crónica en los niños menores de cinco años analizados fue de 19,8\% (IC 95\%: 18,1-21,6). En cuanto a violencia contra la mujer ejercida por la pareja, la prevalencia fue de 30,1\% para violencia emocional (IC 95\%: 28,4-31,8), 6,9\% para violencia sexual (IC 95\%: 6-7,8) y dentro de las categorías de violencia física $20,2 \%$ (IC 95\%: 18,6-21,7) sufrió solo de violencia leve y $14,6 \%$ (IC 95\%: 12,7-15,4) severa.

La edad media de las madres fue de 30,7 años y no varió entre las que tenían o no un hijo con desnutrición $(p=0,644)$. La mayoría tenia secundaria completa $(54,8 \%)$ y evidenciamos una relación inversa entre desnutrición y nivel educativo $(p<0,001)$, yendo de $52,1 \%$ en madres

Tabla 1. Descripción y análisis univariado de covariables de la madre, pareja, del hogar y desnutrición crónica en hijos menores de cinco años en Perú 2011

\begin{tabular}{|c|c|c|c|c|c|}
\hline Variable & $\mathbf{N}$ & $(\%)$ & Desnutrición crónica (\%) & RP (IC 95\%) & $p$ \\
\hline Edad de la madre & 5154 & $(30,7)$ & $(30,6)^{*}$ & $1,00(0,97-1,01)$ & 0,644 \\
\hline \multicolumn{6}{|l|}{ Nivel educativo de la madre } \\
\hline Sin educación & 179 & $(3,5)$ & $(52,1)$ & 1 & $<0,001^{a}$ \\
\hline Primaria & 1767 & $(35,0)$ & $(37,7)$ & $0,72(0,60-0,87)$ & \\
\hline Secundaria & 2770 & $(54,8)$ & $(10,9)$ & $0,21(0,17-0,26)$ & \\
\hline Superior & 336 & $(6,7)$ & $(4,2)$ & $0,08(0,04-0,15)$ & \\
\hline \multicolumn{6}{|l|}{ Uniones conyugales } \\
\hline Una & 4527 & $(88,0)$ & $(19,8)$ & 1 & 0,964 \\
\hline Más de una & 620 & $(12,0)$ & $(19,9)$ & $1,01(0,82-1,23)$ & \\
\hline \multicolumn{6}{|l|}{ Agresión durante el embarazo } \\
\hline Agredida por cualquiera & 606 & $(11,8)$ & $(19,5)$ & 1 & 0,895 \\
\hline No agredida & 4535 & $(88,2)$ & $(19,8)$ & $1,01(0,82-1,25)$ & \\
\hline \multicolumn{6}{|l|}{ Madre trabaja } \\
\hline No & 1910 & $(37,1)$ & $(18,1)$ & 1 & 0,049 \\
\hline Sí & 3237 & $(62,9)$ & $(21,0)$ & $1,16(1,00-1,34)$ & \\
\hline \multicolumn{6}{|l|}{ Madre trabaja fuera de casa } \\
\hline No & 655 & $(17,2)$ & $(14,1)$ & 1 & $<0,001$ \\
\hline $\mathrm{Si}$ & 3151 & $(82,8)$ & $(22,1)$ & $1,57(1,22-2,01)$ & \\
\hline Edad en el primer matrimonio & 5147 & $(20,5)$ & $(19,2)^{*}$ & $0,94(0,92-0,95)$ & $<0,001$ \\
\hline \multicolumn{6}{|c|}{ Hirió al esposo en ausencia de agresión } \\
\hline No & 4791 & $(93,1)$ & $(20,7)$ & 1 & $<0,001$ \\
\hline Sí & 356 & $(6,9)$ & $(9,0)$ & $0,43(0,30-0,62)$ & \\
\hline Edad de la pareja & 4699 & $(30,6)$ & $(34,4)^{*}$ & $1,00(0,99-1,00)$ & 0,398 \\
\hline \multicolumn{6}{|l|}{ Nivel educativo pareja } \\
\hline Sin educación & 63 & $(1,2)$ & $(42,0)$ & 1 & $<0,001^{a}$ \\
\hline Primaria & 1383 & $(27,0)$ & $(36,9)$ & $0,88(0,62-1,25)$ & \\
\hline Secundaria & 3228 & $(63,0)$ & $(15,7)$ & $0,37(0,26-0,53)$ & \\
\hline Superior & 450 & $(8,8)$ & $(4,3)$ & $0,10(0,06-0,18)$ & \\
\hline \multicolumn{6}{|l|}{ Frecuencia que pareja se embriaga } \\
\hline Nunca & 460 & $(11,9)$ & $(12,6)$ & 1 & $<0,001$ \\
\hline Frecuentemente & 356 & $(9,2)$ & $(23,2)$ & $1,84(1,31-2,58)$ & \\
\hline Algunas veces & 3039 & $(78,8)$ & $(20,3)$ & $1,61(1,22-2,11)$ & \\
\hline \multicolumn{6}{|l|}{ Esposo vive en casa } \\
\hline Vive con la madre & 4457 & $(94,9)$ & $(20,1)$ & 1 & 0,454 \\
\hline Vive fuera de casa & 242 & $(5,1)$ & $(17,8)$ & $0,88(0,64-1,22)$ & \\
\hline Edad del jefe del hogar & 5147 & $(39,9)$ & $(37,5)^{\star}$ & $0,98(0,98-0,99)$ & $<0,001$ \\
\hline \multicolumn{6}{|l|}{ Residencia } \\
\hline Urbano & 2903 & $(56,4)$ & $(10,6)$ & 1 & $<0,001$ \\
\hline Rural & 2244 & $(43,6)$ & $(38,0)$ & $3,60(2,98-4,35)$ & \\
\hline \multicolumn{6}{|l|}{ Índice de riqueza } \\
\hline Muy pobre & 1537 & $(29,9)$ & $(45,4)$ & 1 & $<0,001^{a}$ \\
\hline Pobre & 1371 & $(26,6)$ & $(22,9)$ & $0,51(0,44-0,58)$ & \\
\hline Medio & 1145 & $(22,3)$ & $(10,8)$ & $0,23(0,19-0,30)$ & \\
\hline Rico & 726 & $(14,1)$ & $(6,5)$ & $0,14(0,09-0,22)$ & \\
\hline Muy rico & 368 & $(7,2)$ & $(2,1)$ & $0,05(0,02-0,10)$ & \\
\hline
\end{tabular}

*(media), $a=p$ de tendencia 
sin educación a 4,2\% en madres con educación superior. Además, $62,9 \%$ de las madres reportó trabajar, de estas $17,2 \%$ dijo hacerlo fuera de casa, estas dos condiciones mostraron reducción en la prevalencia de desnutrición de 16 y $57 \%$ respectivamente $(p=0,049$ y $p<0,001)$. Asimismo, por cada año a más que la madre tuvo al contraer matrimonio por la primera vez se redujo la prevalencia de desnutrición crónica en 6,0\% (RP 0,94\%; IC 95\%: 0,92-0,95) (Tabla 1).

La edad media de la pareja fue de 34,6 años, pero no mostró afectar las prevalencias de desnutrición $(p=0,398)$. La mayoría tenia secundaria completa $(63,0 \%)$ y al igual que con las madres se vio una tendencia inversa entre nivel educativo y prevalencia de desnutrición $(p<0,001)$. Por otro lado, $78,8 \%$ de las parejas se embriagaban algunas veces y $9,2 \%$ frecuentemente, lo cual se mostró asociado a un aumento en las prevalencias de desnutrición $(p<0,001)$. La edad del jefe del hogar estuvo asociada a una reducción de $2,0 \%$ en la prevalencia de desnutrición por cada año a más que este tenía (RP 0,98\%, IC 95\%: 0,98-0,99). Niños de zonas rurales $(43,6 \%)$ mostraron una prevalencia de desnutrición 3,6 veces mayor que los urbanos $(p<0,001)$ y hubo una tendencia a disminuir la prevalencia de desnutrición al aumentar el índice de riqueza $(p<0,001)$ (Tabla 1).
En los hijos, la edad media fue de 30,6 meses y no se mostró asociada a desnutrición $(p=0,128)$. El $50,2 \%$ eran mujeres y mostraron una prevalencia de desnutrición 14,0\% menor que los hombres $(p=0,038)$. El orden de nacimiento $(p<0,001)$, peso al nacer $(p<0,001)$ y tiempo de gestación en el primer control $(p<0,001)$, mostraron una asociación positiva con desnutrición, por otro lado número de visitas prenatales $(p<0,001)$, parto profesional $(p<0,001)$, uso de biberón $(p<0,001)$ y control posnatal a los dos meses $(p<0,001)$, mostraron asociación inversa (Tabla 2).

\section{VIOLENCIA Y DESNUTRICIÓN CRÓNICA}

La presencia de violencia física severa contra las mujeres por parte del compañero actual estuvo asociado de manera significativa con un aumento de la prevalencia de desnutrición crónica en los últimos nacidos vivos menores de cinco años, en el análisis crudo (RP 1,40\%, IC 95\%: 1,18-1,65), atenuándose ligeramente en el análisis ajustado (RP 1,27\%, IC: 95\%: 1,09-1,48). Violencia emocional, física leve o sexual no presentaron asociación con desnutrición crónica (Tabla 3).

Todos los modelos fueron ajustados para índice de riqueza, nivel educativo de la madre, agresión durante

Tabla 2. Descripción y análisis univariado de variables independientes relacionadas al hijo menor de cinco años y desnutrición crónica en Perú 2011

\begin{tabular}{|c|c|c|c|c|c|}
\hline Variable & $\mathbf{N}$ & $(\%)$ & $\begin{array}{c}\text { Desnutrición } \\
\text { crónica (\%) }\end{array}$ & RP (IC 95\%) & $p$ \\
\hline Edad en meses & 5147 & $(30,6)^{*}$ & $(29,9)^{*}$ & $1,00(0,99-1,00)$ & 0,128 \\
\hline \multicolumn{6}{|l|}{ Sexo } \\
\hline Masculino & 2565 & $(49,8)$ & $(21,2)$ & 1 & 0,038 \\
\hline Femenino & 2582 & $(50,2)$ & $(18,4)$ & $0,86(0,75-0,99)$ & \\
\hline Orden de nacimiento & 5147 & $(2,7)$ & $(3,5)^{*}$ & $1,21(1,18-1,24)$ & $<0,001$ \\
\hline kg de peso al nacer & 5147 & $(3,8)$ & $(4,2)^{*}$ & $1,11(1,08-1,15)$ & $<0,001$ \\
\hline Meses de gestación primer control & 5147 & $(2,6)$ & $(3,0)^{*}$ & $1,14(1,10-1,17)$ & $<0,001$ \\
\hline \multicolumn{6}{|l|}{ Lugar del parto } \\
\hline Hospital & 2433 & $(48,3)$ & $(11,0)$ & 1 & $<0,001$ \\
\hline Casa & 913 & $(18,1)$ & $(45,6)$ & $4,13(3,40-5,01)$ & \\
\hline Centro o puesto de salud & 1338 & $(26,6)$ & $(26,4)$ & $2,39(1,95-2,92)$ & \\
\hline Clínica o médico privado & 279 & $(5,5)$ & $(7,9)$ & $0,72(0,43-1,19)$ & \\
\hline Otros & 75 & $(1,5)$ & $(24,7)$ & $2,24(1,37-3,65)$ & \\
\hline \multicolumn{6}{|l|}{ Parto profesional } \\
\hline Sí & 4186 & $(81,3)$ & $(15,6)$ & $0,37(0,32-0,42)$ & \\
\hline \multicolumn{6}{|l|}{ Control posnatal a los dos meses } \\
\hline No & 134 & $(2,7)$ & $(55,0)$ & 1 & $<0,001$ \\
\hline Sí & 4872 & $(97,3)$ & $(19,1)$ & $0,35(0,29-0,42)$ & \\
\hline \multicolumn{6}{|l|}{ Duración de lactancia materna } \\
\hline $6-24$ meses & 2593 & $(51,5)$ & $(19,0)$ & 1 & $<0,001$ \\
\hline$>24$ meses & 315 & $(6,3)$ & $(20,8)$ & $1,09(0,83-1,44)$ & \\
\hline$<6$ meses & 144 & $(2,9)$ & $(4,9)$ & $0,25(0,13-0,50)$ & \\
\hline Aun lacta & 1930 & $(38,3)$ & $(22,1)$ & $1,16(1,01-1,33)$ & \\
\hline Nunca & 55 & $(1,1)$ & $(20,1)$ & $1,06(0,58-1,94)$ & \\
\hline \multicolumn{6}{|l|}{ Uso de biberón } \\
\hline No & 3573 & $(71,3)$ & $(22,9)$ & 1 & $<0,001$ \\
\hline Sí & 1439 & $(28,7)$ & $(12,9)$ & $0,56(0,47-0,67)$ & \\
\hline
\end{tabular}

*(media) 
Tabla 3. Análisis crudo y ajustado entre violencia contra la mujer y desnutrición crónica en hijos menores de cinco años y prevalencia de desnutrición crónica para cada tipo de violencia, Perú 2011

\begin{tabular}{|c|c|c|c|c|}
\hline Variable & $\mathrm{n} / \mathrm{N}$ & \% Desnutrición crónica (IC 95\%) & RP crudo (IC 95\%) & RP ajustado (IC 95\%)* \\
\hline \multicolumn{5}{|c|}{ Violencia emocional } \\
\hline No & $3544 / 5075$ & $20,3 \%(18,1-22,6)$ & 1 & 1 \\
\hline Sí & $1531 / 5075$ & $18,6 \%(16,2-20,9)$ & $0,91(0,79-1,05)$ & $1,03(0,91-1,17)$ \\
\hline \multicolumn{5}{|l|}{ Violencia sexual } \\
\hline No & $4685 / 5075$ & $20,3 \%(18,1-22,6)$ & 1 & 1 \\
\hline Sí & $390 / 5075$ & $22,7 \%(17,7-27,7)$ & $1,16(0,92-1,47)$ & $1,09(0,86-1,38)$ \\
\hline \multicolumn{5}{|l|}{ Violencia física } \\
\hline No & $3180 / 5001$ & $19,7 \%(17,6-21,8)$ & 1 & 1 \\
\hline Leve & $1016 / 5001$ & $14,5 \%(11,8-17,2)$ & $0,74(0,60-0,89)$ & $0,91(0,76-1,10)$ \\
\hline Severa & $805 / 5001$ & $27,6 \%(23,6-31,6)$ & $1,40(1,18-1,65)$ & $1,27(1,09-1,48)$ \\
\hline
\end{tabular}

*Modelo ajustado para índice de riqueza, nivel educativo de la madre, agresión durante el embarazo, edad de la madre, edad en el primer matrimonio, edad del jefe del hogar, residencia en zona rural, sexo del niño, orden nacimiento, peso al nacer y lugar de parto. Violencia física fue además ajustada para uso de biberón y edad del niño.

el embarazo, edad de la madre, edad en el primer matrimonio, edad del jefe del hogar, residencia en zona rural, sexo del niño, orden nacimiento, peso al nacer y lugar de parto. Violencia física fue además ajustada para uso de biberón y edad del niño.

\section{DISCUSIÓN}

Encontramos que de los diferentes tipos de violencia contra la mujer ejercida por la pareja intima, la violencia física severa contra la mujer estuvo asociada con un aumento en la prevalencia de desnutrición crónica en sus hijos menores de cinco años.

Al igual que nosotros, diferentes autores alrededor del mundo han encontrado asociaciones similares a la nuestra (18-22). En 2008 Ackerson, en una encuesta similar a la ENDES en India (Indian National Family Health Survey), encontró que la violencia doméstica física contra la mujer, por parte de cualquier miembro de la familia, ejercida una o más veces en el último año, aumentaba las chances de desnutrición crónica en sus hijos ${ }^{(18)}$. Cabe resaltar que nosotros evaluamos únicamente la violencia ejercida por la pareja intima, sin contar el número de episodios y Ackerson no llegó a investigar otros tipos de violencia como sexual y emocional. En otro estudio de diferentes DHS, en Egipto, Honduras, Kenia, Malawi y Ruanda, Rico et al. (2011) encontraron que existía una asociación entre violencia física ejercida por la pareja y desnutrición crónica, cosa que no observaron con violencia sexual. Cabe resaltar que esta asociación solo se observó en las madres en Kenia y sus hijos, sin ser reproducido en los otros países ${ }^{(20)}$, lo que nos hace pensar que el contexto donde ocurre la violencia es importante para la asociación de interés. Por otro lado, los hallazgos de Sobkoviak et al. (2012) indican que únicamente la violencia sexual, y no la física o emocional, estuvieron asociados a una mayor oportunidad de desnutrición crónica en niños menores de cinco años ${ }^{(19)}$, contrario a lo que los autores anteriores y nosotros encontramos. Sin embargo Asling-Monemi et al. (2009) encontraron una relación entre cualquier tipo de violencia doméstica (física, emocional o sexual) y disminución del escore $Z$ de talla para la edad a los 24 meses de vida, así como en el crecimiento (21); aun así, estos resultados fueron medidos de forma continua y solo en niños de 24 meses, lo que los hace de difícil comparación con nuestros resultados.

Los caminos causales que podrían explicar la relación entre violencia severa ejercida por la pareja íntima y desnutrición son múltiples ${ }^{(5)}$. Existe evidencia que muestra que mujeres víctimas de violencia ejercida por la pareja presentan pobre autorreporte de salud física, prevalencias mayores de trastornos mentales y de abuso de drogas y alcohol ${ }^{23)}$. Asimismo, víctimas durante el período gestacional presentan mayor oportunidad de hijos con bajo peso al nacer, parto pretérmino, muerte en el periodo neonatal y un patrón de lactancia menos adecuado ${ }^{(23)}$. Todos estos factores, que pueden ser productos de la violencia, se han visto relacionados con malnutrición en niños ${ }^{(14,24-27)}$. Por otro lado, el estrés al que están sometidos los niños que viven en estos hogares puede llevar a estados de alta actividad inflamatoria y prooxidante, como fue observado en 2010 por Houphouet ${ }^{(28)}$, donde la malnutrición estuvo asociada con estado inflamatorio, indicado por aumento de proteína $\mathrm{C}$ reactiva, la cual, además, se acompañaba de depleción proteica, especialmente de albúmina, lo que podría ser un factor contribuyente a la desnutrición $(29,30)$. 
Es importante resaltar ciertas limitaciones y fortalezas del presente estudio. Dado el diseño y la forma cómo fueron hechas las preguntas en la ENDES, no tenemos la capacidad para definir una relación causal, primero por no poder evaluar temporalidad, la violencia fue autorreportada para los últimos 12 meses, y la desnutrición crónica no es un evento que se inicia necesariamente en ese mismo periodo. Es más, la desnutrición crónica, como problema multicausal, tiene sus inicios en etapas tempranas de la vida $(14,27)$. Si bien en nuestro caso el episodio de violencia ejercida por la pareja se circunscribe a los últimos 12 meses, es posible que la misma haya iniciado antes, por tanto, es otra limitación del estudio no poder evaluar el tiempo durante el cual la mujer fue víctima de violencia. En nuestro estudio no podemos establecer si la violencia física severa, reportada de manera aislada en el último año, es en sí lo que produce la desnutrición. Sin embargo, consideramos que esta experiencia de vida nos da pie a un indicador del tiempo y/o número de episodios de violencia experimentados, pues se sabe que la violencia severa ejercida por la pareja íntima se da en mujeres que han sufrido mayor tiempo de abusos, siendo los primeros episodios leves y aumentando de intensidad con el transcurso de los años ${ }^{(1)}$. Asimismo, otros criterios de causalidad como dosis-respuesta no pudieron ser explorados, el efecto acumulado de la violencia a través de los años o inclusive el número de episodios de violencia en el último año, nos habría dado una mayor fuerza para pensar en una relación causal. Sin embargo, consideramos que tenemos otros criterios que pueden tomarse en consideración, como la plausibilidad de la relación ${ }^{(5)}$, coherencia, al comparar con diferentes estudios que ha encontrado resultados similares a los nuestros y analogía ${ }^{(18-22)}$. En tal sentido, este estudio propone a la violencia contra la mujer ejercida por la pareja íntima, como una variable a considerar en la problematización de la desnutrición crónica, sin poder establecer una relación causal.

También es importante resaltar que algunas posibles variables confusoras, como salud mental de la madre y de la pareja, así como la frecuencia de consumo de alcohol, no fueron evaluadas en este estudio por falta de disponibilidad en la base de datos, y porque esta última tenía pérdidas de información no aleatoria y podría haber sesgado nuestros resultados. Sin embargo, consideramos que aún no es claro si la salud mental podría considerarse un confusor de la relación o un mediador del efecto de la violencia sobre la desnutrición ${ }^{(5)}$, por lo que estudios que profundicen ese tema son necesarios para poder dar conclusiones más contundentes.
Asimismo, cabe resaltar que los datos de violencia fueron tomados de una submuestra y se tuvo que elegir una subpoblación de la misma (solo el último hijo) para hacer los análisis. Al hacer las comparaciones con las madres y niños no incluidos en el análisis, notamos algunas diferencias, por lo que la capacidad para extrapolación de resultados podría verse afectada. Aun así, consideramos que la validez interna del estudio es adecuada, existe plausibilidad para pensar que esto mismo podría acontecer en poblaciones similares $\mathrm{y}$, por tanto, los resultados deben ser tomados en consideración e intentar ser reproducidos en estudios con contextos distintos. Por otro lado, cabe resaltar que este es un estudio de base poblacional, con más de cinco mil observaciones y, hasta donde conocemos, es uno de los primeros estudios, en el contexto Latinoamérica, donde se explora esta relación.

Concluimos que nuestro estudio encontró que la violencia física severa ejercida contra la mujer, en los últimos 12 meses, por la pareja íntima, está asociada con un aumento de la prevalencia de desnutrición crónica en sus hijos menores de cinco años. Otros estudios han encontrado resultados similares a los nuestros, pero esta relación requiere continuas exploraciones en diferentes contextos culturales, tomando en consideración variables de salud mental, ya sea como posibles confusores o mediadores de efecto. Sin embargo, consideramos que existe evidencia para pensar que la violencia física severa contra la mujer puede tener efectos a largo plazo sobre sus hijos menores de cinco años, por lo que consideramos que políticas públicas con el objetivo de combatir la violencia contra la mujer, no solo por la salud de las víctimas sino también la de sus hijos.

Contribuciones de autoría: concepción y diseño del trabajo: PRG, JJM; análisis e interpretación de datos: PRG, CLdM, JJM; redacción del manuscrito: PRG, CLdM, JJM; revisión crítica del manuscrito: PRG, CLdM, JJM; aprobación de su versión final: PRG, CLdM, JJM; asesoría estadística: CLdM, JJM; asesoría técnica o administrativa: JJM.

Fuentes de financiamiento: los autores están afiliados a CRONICAS Centro de Excelencia en Enfermedades Crónicas de la UPCH el cual es financiado por el National Heart, Lung and Blood Institute (NHLBI), National Institute of Health (NIH), Department of Health and Human Services, mediante contrato No. HHSN268200900033C.

Conflictos de interés: los autores declaran no tener conflictos de interés. 


\section{REFERENCIAS BIBLIOGRÁFICAS}

1. Bardales $\mathrm{O}$, Vásquez $\mathrm{H}$. Feminicidio bajo la lupa: violencia hacia la mujer, feminicidio, servicios de atención de la violencia. Lima: Ministra de la Mujer y Poblaciones Vulnerables; 2012.

2. García-Moreno C, Jansen HAFM, Ellsberg M, Heise L, Watts C. WHO Multi-country Study on Women's Health and Domestic Violence against Women. Geneva: WHO; 2005.

3. Klevens J.Violencia física contra la mujer en Santa Fe de Bogotá: prevalencia y factores asociados. Rev Panam Salud Publica. 2001;9(2):78-83.

4. Miranda MP, de Paula CS, Bordin IA. Violência conjugal física contra a mulher na vida: prevalência e impacto imediato na saúde, trabalho e família. Rev Panam Salud Publica. 2010;27(4):300-8.

5. Yount KM, DiGirolamo AM, Ramakrishnan U. Impacts of domestic violence on child growth and nutrition: a conceptual review of the pathways of influence. Soc Sci Med. 2011;72(9):153454. doi: 10.1016/j.socscimed.2011.02.042.

6. Wuest J, Merritt-Gray M, Ford-Gilboe M, Lent B, Varcoe C, Campbell JC. Chronic pain in women survivors of intimate partner violence. J Pain. 2008;9(11):104957. doi: 10.1016/j.jpain.2008.06.009.

7. Woods SJ, Hall RJ, Campbell JC, Angott DM. Physical health and posttraumatic stress disorder symptoms in women experiencing intimate partner violence. J Midwifery Womens Health. 2008;53(6):538-46. doi: 10.1016/j. jmwh.2008.07.004.

8. Kally Z, Cumella EJ. 100 midlife women with eating disorders: a phenomenological analysis of etiology. J Gen Psychol. 2008;135(4):359-77. doi: 10.3200/ GENP.135.4.359-378.

9. Alsaker K, Moen BE, Nortvedt MW, Baste V. Low health-related quality of life among abused women. Qual Life Res. 2006;15(6):959-65.

10. Fried LE, Cabral H, Amaro H, Aschengrau A. Lifetime and during pregnancy experience of violence and the risk of low birth weight and preterm birth.J Midwifery Womens Health. 2008;53(6):522-8. doi: 10.1016/j.jmwh.2008.07.018.

11. Neggers Y, Goldenberg R, Cliver S, Hauth $\mathrm{J}$. Effects of domestic violence on preterm birth and low birth weight. Acta Obstet Gynecol Scand. 2004;83(5):455-60.

12. Núñez-Rivas HP, Monge-Rojas R, GríosDávila C, Elizondo-Ureña AM, Rojas-
Chavarría A. La violencia física, psicológica, emocional y sexual durante el embarazo: riesgo reproductivo predictor de bajo peso al nacer en Costa Rica. Rev Panam Salud Publica. 2003;14(2):75-83.

13. Sanchez SE, Alva AV, Diez Chang G, Qiu C, Yanez D, Gelaye B, et al. Risk of spontaneous preterm birth in relation to maternal exposure to intimate partner violence during pregnancy in Peru. Matern Child Health J. 2013;17(3):485-92. doi: 10.1007/s10995-012-1012-0.

14. Santos IS, Matijasevich A, Domingues MR, Barros AJ, Victora CG, Barros FC. Late preterm birth is a risk factor for growth faltering in early childhood: a cohort study. BMC Pediatr. 2009;9:71. doi: 10.1186/1471-2431-9-71.

15. Instituto Nacional de Estadística e Informática (INEI). Encuesta Demográfica y de Salud Familiar. Diseño muestral. ENDES. Lima: INEI; 2008.

16. World Health Organization (WHO). WHO Global Database on Child Growth and Malnutrition [Internet]. Geneva: WHO; 2006 [citado el 21 de junio del 2013]. Disponible en: http://www.who. int/nutgrowthdb/en/

17. Barros AJ, Hirakata VN. Alternatives for logistic regression in cross-sectional studies: an empirical comparison of models that directly estimate the prevalence ratio. BMC Med Res Methodol. 2003;3:21.

18. Ackerson LK, Subramanian SV. Domestic violence and chronic malnutrition among women and children in India. Am J Epidemiol. 2008;167(10):1188-96. doi: 10.1093/aje/kwn049.

19. Sobkoviak RM, Yount KM, Halim N. Domestic violence and child nutrition in Liberia. Soc Sci Med. 2012;74(2):103-11. doi: 10.1016/j.socscimed.2011.10.024.

20. Rico E, Fenn B, Abramsky T, Watts C. Associations between maternal experiences of intimate partner violence and child nutrition and mortality: findings from Demographic and Health Surveys in Egypt, Honduras, Kenya, Malawi and Rwanda. J Epidemiol Community Health. 2011;65(4):360-7. doi: 10.1136/ jech.2008.081810.

21. Asling-Monemi K, Naved RT, Persson LA. Violence against women and the risk of fetal and early childhood growth impairment: a cohort study in rural Bangladesh. Arch Pediatr Adolesc Med. 2009;163(10):9316. doi: 10.1001/archpediatrics.2009.167.
22. Hasselmann MH, Reichenheim ME. Parental violence and the occurrence of severe and acute malnutrition in childhood. Paediatr Perinat Epidemiol. 2006;20(4):299-311.

23. Sarkar NN. The impact of intimate partner violence on women's reproductive health and pregnancy outcome. J Obstet Gynaecol. 2008;28(3):266-71. doi: 10.1080/01443610802042415.

24. Patel V, DeSouza N, Rodrigues $M$. Postnatal depression and infant growth and development in low income countries: a cohort study from Goa, India. Arch Dis Child. 2003;88(1):34-7.

25. Anoop S, Saravanan B, Joseph A, Cherian A, Jacob KS. Maternal depression and low maternal intelligence as risk factors for malnutrition in children: a community based case-control study from South India. Arch Dis Child. 2004;89(4):325-9

26. Rao KN, Begum S, Venkataramana V, Gangadharappa N. Nutritional neglect and physical abuse in children of alcoholics. Indian J Pediatr. 2001;68(9):843-5.

27. Cobayashi F, Augusto RA, Lourenco BH, Muniz PT, Cardoso MA. Factors associated with stunting and overweight in Amazonian children: a population-based, cross-sectional study. Public Health Nutr. 2013 Mar 4:1-10. [Epub ahead of print]

28. Houphouet FY, Yapo A, Dodehe Y, Ahiboh H, Nguessan JD, Hauhouot AM, et al. Effet des Malnutritions Mineure et Moderee sur les Proteines Immunitaires, Inflammatoires et Nutritionnelles Chez L'Enfant en Cote D'Ivoire. Mali Med. 2010;25(2):13-6.

29. Hapuarachchi JR, Chalmers $\mathrm{AH}$, Winefield AH, Blake-Mortimer JS. Changes in clinically relevant metabolites with psychological stress parameters. Behav Med. 2003;29(2):52-9.

30. Irie M, Asami $S$, Nagata $S$, Miyata $M$, Kasai H. Relationships between perceived workload, stress and oxidative DNA damage. Int Arch Occup Environ Health. 2001;74(2):153-7.

Correspondencia: Christian Loret de Mola

Dirección: CRONICAS Centro de Excelencia en Enfermedades Crónicas, Universidad Peruana Cayetano Heredia. Av. Armendáriz 497, 2 do Piso, Miraflores, Lima 18, Perú

Teléfono: +5112416978

Correo electrónico:christian.loret.de.mola.z@upch.pe 